\title{
Evaluation Of Progesterone Level And Endometrial Pattern As Predictors For Pregnancy In Intra-Cytoplasmic Sperm Injection Original Cycles Article
}

\author{
W. S. Elgazayerli
}

Department of Obstetrics and Gynecology, Alexandria University

\begin{abstract}
Background: Intra cytoplasmic sperm injection (ICSI) is one of the effective treatments in women with fertility problems; however, there are no approved predictors for pregnancy till the present moment. We aimed to evaluate the role of progesterone and endometrium pattern in predicting pregnancy in ICSI cycles.

Patients and Methods: The study included 200 women who were treated in Al-Shatby Hospital in (ICSI) Unit from January 2017 to October 2018. They underwent one super ovulation protocol which was gonadotrophin in which releasing hormone progesterone was measured in the $14^{\text {th }}$ day; while ultrasound was performed in the 3rd day and in the day of HCG administration.

Results and Conclusion: Progesterone with a cutoff point of $27 \mathrm{ng} / \mathrm{mol}$ is a significant predictor of pregnancy in ICSI cycle. However, neither endometrial thickness nor pattern was a significant predictor of pregnancy.
\end{abstract}

Key Words: Endometrial pattern, ICSI, progesterone

Received: 3 May 2019, Accepted: 12 June 2019

Corresponding Author: W. S. Elgazayerli, MD, Department of Obstetrics and Gynecology, Alexandria University, Egypt, Tel.: +2 01224283287, E-mail: welgazayerli@yahoo.com

ISSN: 2090-7265, August 2019, Vol. 9, No. 3

\section{INTRODUCTION}

Infertility is a common problem with a huge social, economic and psychological burden. ${ }^{[1]}$ The effective solution of this problem; namely, assisted reproductive technology, has been used for years. One of the common techniques of assisted reproductive technology is intra cytoplasmic sperm injection (ICSI). ${ }^{[2]}$ ICSI protocols have continued to evolve with efforts to improve outcomes. As a result, treatment success may be related to certain procedural factors including number of embryos transferred and stage at which they are transferred. ${ }^{[2]}$ Nevertheless, finding a sensitive predictor for pregnancy remains an issue.

Estrogen and progesterone are considered essential in the reproduction process. Roles of progesterone include implantation and pregnancy maintenance. It has been used in ICSI for luteal support after embryo transfer (ET) and early pregnancy maintenance in cases of positive $\beta$-hCG check ${ }^{[3]}$. It was suggested that progesterone plays a role in the modulation of maternal immune response $\mathrm{e}^{[4]}$, reduction of uterine contractility ${ }^{[5]}$ and improvement of utero-placental circulation ${ }^{[6]}$. These data suggests a merit of progesterone in prediction of pregnancy in ICSI cycles.

In addition to embryo quality, the receptivity of the endometrium plays an important role in clinical outcome. Sonography is a noninvasive and simple method that has been used to evaluate endometrial receptivity. Several sonographic parameters have been evaluated. These included endometrial thickness, endometrial pattern, endometrial volume and endometrial and sub-endometrial blood flow.

The aim of this study is to compare between progesterone and endometrial pattern in prediction of pregnancy in ICSI cycles.

\section{MATERIAL AND METHODS}

The study was approved by the ethics committee of Alexandria University.

\section{Subjects}

This is a prospective study that randomly included 200 women who were treated in Al-Shatby Hospital in ICS Unit from January 2017 to October 2018. Only those who completed the ICSI-embryo transfer (ET) cycle and had a pregnancy test in our laboratory 14 days post-oocyte retrieval were included. Women who had a blood test either in another laboratory or on a different day apart from day 14 or were lost on follow up were excluded. Exclusion criteria also included presence of known endometrial polyp, uterine anomaly and insemination method other than IVF, cycles using donor oocytes or cryopreserved embryos. 
Only one super ovulation protocol was used, which was gonadotrophin, in which releasing hormone $(\mathrm{GnRH})$ agonist protocol. Women began down regulation with buserelin acetate (Shire Pharmaceuticals, Andover, UK) on day 2 or 21 of their cycles for 2 weeks following which they started ovarian stimulation with recombinant follicle stimulating hormone ( $\mathrm{rFSH}$ ) (Puregon, Organon, UK or Gonal-F, Serono, UK). There was close monitoring of the ovarian response in every subject with transvaginal ultrasonography and serum estradiol and luteinizing hormone (LH) measurements.

When at least three follicles, $17 \mathrm{~mm}$ in size, women were administered 10.000 IU of human chorionic gonadotrophin (Pregnyl, Organon, UK or Profasi, Serono, UK) to simulate the LH surge of a natural cycle. They underwent transvaginal ultrasound-guided oocyte recovery (TVOR) $36 \mathrm{~h}$ later under intravenous sedation. That day was defined as day 0 . All women were given a 7-day course of doxycycline afterwards. Oocytes were fertilized single sperm injection (ICSI) and a maximum three embryos were transferred in the uterine cavity 2 or 3 days afterwards.

After ET, all women had luteal phase support with natural progesterone given as a single injection $100 \mathrm{mg}$ (Gestone, Ferring, UK) on the day of ET and then daily $400 \mathrm{mg}$ in the form of a single rectal suppository (Cyclogest 400, Shire Pharmaceuticals, UK) until their pregnancy blood test on day 14 post-TVOR. Women who, on day 14 , had levels of the B-hCG $1 \mathrm{IU} / 1$ were excluded from the study. The rest were followed up either with further blood tests until B-hCG was $1 \mathrm{IU} / 1$ or with serial transvaginal ultrasonography until 8 weeks of gestation when they had a normal pregnancy, or until they were diagnosed with a miscarriage or an ectopic pregnancy. Women with either ectopic pregnancy were also excluded from the study.

\section{Ultrasound}

Endometrial features assessed included endometrial thickness and pattern on day 3 of gonadotrophin stimulation and on the day of HCG administration as well as the change in endometrial thickness from day 3 to day of HCG administration. All were measured by transvaginal $8 \mathrm{MHz}$ ultrasonography with Doppler ultrasound (Mindray DC-6 Expert; Shenzhen, China). Endometrial thickness was measured in a median longitudinal plane of the uterus as the maximum distance between the endometrial-myometrial interfaces of the anterior to the posterior wall of the uterus. Endometrial pattern was classified by a single researcher as A for trilaminar, B for intermediate isoechogenic and $\mathrm{C}$ for homogenous hyperechoic.

\section{Hormonal assay}

Blood samples were drawn on day 14 between 7 and 9 am in standard SST gel tubes (Becton Dickinson, Plymouth, UK) and were allowed to clot before centrifugation at 3000 $\mathrm{g}$ for $10 \mathrm{~min}$ in order to separate the serum. Samples were transferred to the laboratory within $1 \mathrm{~h}$ of collection and analyzed on the same day for progesterone with sequential competitive immunoassay (Immulite, DPC, Los Angeles, $\mathrm{CA}$ ) and B-hCG with microparticle immunoassay (Architect, Abbott laboratories, Illinois, IL). The analytical performance characteristics of the progesterone assay were sensitivity $0.6 \mathrm{nmol} / 1$, inter-assay precision (coefficient of variation $\mathrm{CV}$ ) $6.8 \%$ at $24 \mathrm{nmol} / 1$ and $9 \%$ at $55 \mathrm{nmol} / 1$; while for B-hCG they were $0.1 \mathrm{IU} / 1,4.5 \%$ at $35 \mathrm{IU} / 1$ and $4.1 \%$ at 89 IU/l, respectively. The progesterone results were given to the investigators at the end of the study.

\section{Statistics}

Continuous data were expressed as mean $+\mathrm{SD}$ values or as median and range according to the distribution and were analyzed for differences using the Student's t-test. Categorical data were presented as numbers and chi-squared test was used for statistical comparison of percentages. Binary logistic regression analysis was also carried out and the receiver operating characteristic (ROC) curve was applied to determine the predictive value of endometrial parameters. Discriminant analysis was used to evaluate the predictive ability of all of the endometrial parameters combined. The significance level for all analyses was $\mathrm{P}<0.05$. Statistical analysis was carried out using the Statistical Package for Social Sciences (SPSS Inc, Chicago, IL, USA).

\section{RESULTS}

Table 1 shows that age $(35.8 \pm 4.8)$, BMI $(24.9 \pm 3.1)$ and Oocyte (11) number was significantly higher in non-pregnant women than in pregnant women (30.6 \pm 4.4), (21.6 \pm 2.6$)$ and (10), respectively. Nevertheless, endometrial thickness at the day of HCG delivery $(10.9 \pm 2.1)$, days of stimulation $(11.2 \pm 2.3)$ and progesterone (422) were significantly higher in pregnant women than in non-pregnant women (10.2 \pm 2.1$)$, $(11.0 \pm 2.3)$ and (27), respectively.

Table 1: Comparison between pregnant and non-pregnant cohorts

\begin{tabular}{lcccc}
\hline Variable & $\begin{array}{c}\text { All patients } \\
\mathrm{N}=191\end{array}$ & $\begin{array}{c}\text { Pregnant } \\
\mathrm{N}=101\end{array}$ & $\begin{array}{c}\text { Non-preg } \\
\mathrm{N}=90\end{array}$ & $p$-value \\
\hline Age & $31.2 \pm 4.6$ & $30.6 \pm 4.4$ & $35.8 \pm 4.8$ & $<0.001$ \\
BMI & $21.73 \pm 2.87$ & $21.6 \pm 2.6$ & $24.9 \pm 3.1$ & $<0.001$ \\
Oocyte & 10 & 9 & 11 & $<0.001$ \\
Days of stim & $11.1 \pm 2.3$ & $11.2 \pm 2.3$ & $11.0 \pm 2.3$ & $<0.001$ \\
End T d3 & $6.0 \pm 1.5$ & $5.9 \pm 1.5$ & $6.1 \pm 1.6$ & 0.008 \\
End T dH & $10.4 \pm 2.1$ & $10.9 \pm 2.1$ & $10.2 \pm 2.1$ & $<0.001$ \\
End P A \% & $40 \%$ & $22 \%$ & $18 \%$ & $<0.001$ \\
End P B $\%$ & $32 \%$ & $19 \%$ & $13 \%$ & $<0.001$ \\
End P C $\%$ & $28 \%$ & $14 \%$ & $14 \%$ & $<0.001$ \\
Progesterone & 201 & 430 & 27 & $<0.001$ \\
\hline
\end{tabular}


Table 2: Binary logistic regression (Clinical pregnancy served as dependent variable); Method $=$ enter.

\begin{tabular}{lcc}
\hline variable & OR & P-value \\
\hline Age & -0.047 & $<0.001$ \\
BMI & 0.652 & 0.002 \\
End T d3 & 1.010 & 0.51 \\
End T dH & 1.107 & 0.07 \\
End P & 1.243 & 0.06 \\
Progesteron & -0.040 & $<0.001$ \\
\hline
\end{tabular}

progesterone was highly sensitive $(91 \%)$ in prediction of clinical pregnancy in ICSI cycles.

This was in complete accordance with the recent study of Kim et al.$^{[8]}$ who aimed to evaluate the predictive value of the progesterone level at the beta-human chorionic gonadotropin ( $\beta$-hCG) check day for ongoing pregnancy maintenance. They concluded that progesterone level at 14 days after oocyte retrieval can be a good predictive marker for ongoing pregnancy maintenance. However, they used a slightly lower cutoff than the current study ( $25 \mathrm{vs} 28 \mathrm{ng} / \mathrm{mol}$ ). They also reported a slightly lower sensitivity ( $87 \%$ vs $91 \%)$.

Table 3: Sensitivity of progesterone at a cut off $>29(\mathrm{ng} / \mathrm{ml})$

\begin{tabular}{|c|c|c|c|c|c|c|c|}
\hline & AUC & $\mathrm{p}$ & $95 \%$ C.I & Cut off & Sensitivity & PPV & $N P V$ \\
\hline Progesterone $(\mathrm{ng} / \mathrm{ml})$ & $0.668^{*}$ & $0.025^{*}$ & $0.527-0.809$ & $>29$ & 91.0 & 69.2 & 64.7 \\
\hline
\end{tabular}

classified as endometrium pattern A; while $32 \%$ were classified as pattern B and $28 \%$ were classified as pattern C. The percentage of patients classified as endometrial pattern A in pregnant and non-pregnant women was $22 \%$ and $18 \%$, respectively, while the percentage of women classified as pattern B was $19 \%$ and $13 \%$, respectively, and percentage of women classified as pattern $\mathrm{C}$ was $14 \%$ in both pregnant and non-pregnant women, respectively. There were significant statistical differences between pregnant and non-pregnant women as regards to endometrial pattern.

Table 2 portrays the binary logistic regression of different parameters. Parameters which were significantly correlated with clinical pregnancy as a dependent variable were maternal age $(p<0.001)$, maternal BMI $(p=0.002)$ and progesterone $(p<0.001)$. Neither endometrial pattern $(p=0.06)$ nor endometrial thickness $(p=0.07)$ was significantly correlated to clinical pregnancy.

The agreement of progesterone in relation to clinical pregnancy is illustrated in table 3. The sensitivity of progesterone in prediction of clinical pregnancy with a cutoff more than $29 \mathrm{ng} / \mathrm{ml}$ was $89 \%$.

\section{DISCUSSION}

Although serum progesterone has been advocated to be the single most powerful predictor of pregnancy outcome in spontaneous conceptions ${ }^{[7]}$, there is very conflicting evidence when this is tested in $\mathrm{COH}$ protocols that use progesterone luteal support. We aimed in this study to test the reliability of progesterone versus endometrial pattern in prediction of clinical pregnancy in ICSI cycles.

Our biggest observation was that progesterone was significantly higher in pregnant than non-pregnant women. Using a binary regression analysis model, we found that progesterone was significantly correlated to clinical pregnancy. Moreover, we provided some evidence that
Solomon et al. ${ }^{[9]}$ also agreed to our results as their progesterone level is directly correlated with favorable pregnancy outcome among patients undergoing any type of ART. They also found that discriminatory progesterone value may vary by mode of conception. As regards to ICSI, they found a cutoff not far from the current study $(24.2 \mathrm{ng} / \mathrm{ml})$ which yielded a sensitivity of $75 \%$. It is worth mentioning that, they enrolled a huge study material of 2007 subjects.

Some previous studies have suggested discriminatory progesterone levels as low as 21.4 and as high as $82.5 \mathrm{nmol} / 1$ for predicting pregnancy in ICSI cycles ${ }^{[10-12]}$. The main drive for progesterone production comes from continuing stimulation of the corpora luteal by HCG. It seems that in abnormal pregnancies, the low progesterone values can be explained by the lower B-hCG concentrations found and most likely there is no other independent determining factor. The high progesterone levels found at day 14 in normal pregnancies indicated that endogenous progesterone in viable pregnancies is already sufficient to compensate a possible iatrogenic luteal phase defect caused by the use of GnRH agonists or antagonists. That raises the question about the value of giving additional progesterone after day 14 in early pregnancy. This practice, even though hardly supported by evidence in the literature, is still commonly performed.

As regards to endometrial pattern and endometrial thickness, the present study found that there was significant difference in the endometrial thickness and pattern between pregnant and non-pregnant women. Nevertheless, there were no significant correlation between clinical pregnancy and both endometrial pattern and thickness.

This was consistent with the very recent study of Yang and his colleagues which was conducted in 2018. They aimed to evaluate the combined effect of endometrial thickness and pattern on clinical outcomes in females following in 
vitro fertilization/intracytoplasmic sperm injection and frozen-thawed embryo transfer. They reviewed 1512 cycles and had a conclusion that neither individual nor combined analysis of endometrial thickness and pattern had predicting effects on clinical pregnancy.

Chen et al. ${ }^{[13]}$ also aimed to evaluate the combined effect of endometrial thickness and pattern on clinical outcome in patients undergoing in vitro fertilization/ intra-cytoplasmic sperm injection and embryo transfer. They reviewed a total of 2896 cycle and found that endometrial pattern as a single measurement had no correlation to clinical pregnancy prediction; however, they were different from our study in that they suggested that combined thickness with pattern may be of a value in prediction of pregnancy.

Another study that completely agreed to our results is Zaho et al. which was conducted in 2014. Although it was a retrospective study, they provided some evidence that neither endometrial thickness nor pattern were significant predictors for pregnancy in ICSI cycles as they enrolled a total number of 3319 cycles. Moreover, they found the two parameters insignificant even if combined together as a single predictor.

In reviewing our data, pregnant women have a significantly but slightly thinner endometrial thickness on day 3 of gonadotrophin stimulation than that of nonpregnant women, but the difference is not clinically significant with the results falling within the range of measurement error. A significant difference was observed in endometrial thickness on the day of $\mathrm{HCG}$ administration and the change in endometrial thickness from the third day of gonadotrophin stimulation to the day of $\mathrm{HCG}$ administration between pregnant women and non-pregnant women. This finding indicated that adequate endometrial development is favorable for improved pregnancy rate. However, the regression analysis failed to prove this correlation.

This was in contrary to the finding of Zhang et al. ${ }^{[16]}$ who enrolled one thousand nine hundred thirty-three women undergoing ICSI in their study. They concluded that both endometrial pattern and endometrial thickness were significant independent predictors of pregnancy. In addition to that, they suggested that combining the two parameters together as a single predictor increased the prediction value.

The reason why thinner endometrium were associated with lower rate of pregnancy is explained by Casper et al. ${ }^{[17]}$ that it may be related to oxygen tension. When the thickness measured by ultrasound is $<7 \mathrm{~mm}$, the functional layer is thin or absent and the implanting embryo would be much closer to the spiral arteries and the higher vascularity and oxygen concentrations of the basal endometrium. The high oxygen concentrations near the basal layer could be detrimental compared with the usual low oxygen tension of the surface endometrium.
Jarvela and his colleagues also mismatched our results ${ }^{[18]}$ They aimed to compare sonographic endometrial characteristics in ICSI cycles between women who conceive and those who do not. They concluded that existence of a homogeneous endometrial pattern after FSH stimulation seems to be a prognostic sign of an adverse outcome in IVF, while a triple-line pattern after FSH stimulation and a decrease in endometrial volume appear to be associated with conception. However, the study was conducted on only 35 women which was too small material to provide significant evidence.

\section{Strengthens and Limitations}

The main strength points in the current study are the prospective design and the homogeneity of demographic data of the studied cohort that prevented any bios in the results, while the only weakness point is the relatively small size of the material.

\section{CONCLUSION}

We have concluded that progesterone with a cutoff point of $27 \mathrm{ng} / \mathrm{mol}$ is a significant predictor of pregnancy in ICSI cycle; however, neither endometrial thickness nor pattern was a significant predictor of pregnancy.

\section{CONFLICT OF INTEREST}

There are no conflicts of interest.

\section{REFERENCES}

1. Fertility: Assessment and Treatment for People with Fertility Problems (update). Draft Guidance for Stakeholder Consultation. 2012, London: RCOG Press/National Collaborating Centre for Women's and Children's Health for the National Institute for Health and Clinical Excellence (NICE)

2. Dunn AL, Stafinski T, Menon P. An International Survey of Assisted Reproductive Technologies (ARTs) Policies and the Effects of these Policies on Costs, Utilization, and Health Outcomes. Health Policy. 2014;116:238. doi: 10.1016/j. healthpol.2014.03.006.

3. Kim YJ, Ku SY, Jee BC, Suh CS, Kim SH, Choi YM, et al. Increased responsiveness may be associated with tripronuclear embryos in both conventional in vitro fertilization and intracytoplasmic sperm injection cycles using gonadotropin-releasing hormone agonist long protocols: a self-matched observational study. J Obstet Gynaecol Res. 2011;37(1):37-44. doi: 10.1111/j.1447-0756.2010.01313.x . [PubMed]

4. Canellada A, Alvarez I, Berod L, Gentile T. Estrogen and progesterone regulate the IL- 6 signal 
transduction pathway in antibody secreting cells. J Steroid Biochem Mol Biol. 2008;111(3-5):255-61. doi: 10.1016/j.jsbmb.2008.06.009 . [PubMed]

5. Patil AS, Swamy GK, Murtha AP, Heine RP, Zheng X, Grotegut CA. Progesterone Metabolites Produced by Cytochrome P450 3A Modulate Uterine Contractility in a Murine Model. Reprod Sci. 2015;22(12):157786. doi: 10.1177/1933719115589414 . [PMC free article] [PubMed]

6. Dang Y, Li W, Tran V, Khalil RA. EMMPRINmediated induction of uterine and vascular matrix metalloproteinases during pregnancy and in response to estrogen and progesterone. Biochem Pharmacol. 2013;86(6):734-47. doi: 10.1016/j.bcp.2013.06.030 ; [PMC free article] [PubMed]

7. Elson J, Salim R, Tailor A, Banerjee S, Zosmer N and Jurkovic D 2012 rediction of early pregnancy viability in the absence of an ultrasonicallydetectable embryo. Ultrasound Obstet Gynecol 21,57-61.

8. Kim YJ, Shin JH, Hur JY, Kim H, Ku SY, Suh CS. Predictive value of serum progesterone level on $\beta$-hCG check day in women with previous repeated miscarriages after ICSI. PLoS One. 2017;12(7):e0181229. Published 2017 Jul 14. doi:10.1371/journal.pone.0181229

9. Solomon, H. F., et al. (2008). "Prediction of pregnancy outcome by serum progesterone level after assisted reproductive technology." Fertility and Sterility 90: S231.

10. Elson J, Salim R, Tailor A, Banerjee S, Zosmer N and Jurkovic D (2003)Prediction of early pregnancy viability in the absence of an ultrasonicallydetectable embryo. Ultrasound Obstet Gynecol 21,57-61.

11. Peterson CM, Kreger D, Delgado P and Hung TT (1992) Laboratory andclinical comparison of a rapid versus a classic progesterone radioimmunoassayfor use in determining abnormal and ectopic pregnancies. Am JObstet Gynecol 166,562-566.

12. Yeko TR, Gorrill MJ, Hughes LH, Rodi IA, Buster JE and Sauer MV (1987)Timely diagnosis of early ectopic pregnancy using a single bloodprogesterone measurement. Fertil Steril 48,1048-1050.

13. Yang $\mathrm{W}$, Zhang $\mathrm{T}, \mathrm{Li} \mathrm{Z}$, et al. Combined analysis of endometrial thickness and pattern in predicting clinical outcomes of frozen embryo transfer cycles with morphological good-quality blastocyst: A retrospective cohort study. Medicine (Baltimore). 2018;97(2):e9577.

14. Chen SL, Wu FR, Luo C, et al. Combined analysis of endometrial thickness and pattern in predicting outcome of in vitro fertilization and embryo transfer: a retrospective cohort study. Reprod Biol Endocrinol 2010;8:30.

15. Zhao J, Zhang Q, Wang Y, Li Y. Endometrial pattern, thickness and growth inpredicting pregnancy outcome following 3319 ICSI cycle. Reprod Biomed Online. 2014Sep;29(3):291-8. doi: 10.1016/j. rbmo.2014.05.011. Epub 2014 Jun 13. PubMed PMID: 25070912.

16. Zhang Q, Li Y. The effect of endometrial thickness and pattern measured by ultrasonography on pregnancy outcomes during ICSI cycles. Reprod Biol Endocrinol 2012;10:100.

17. Casper RF. It's time to pay attention to the endometrium. Fertil Steril. 2011;96:519-521. doi: 10.1016/j.fertnstert.2011.07.1096.

18. Jarvela IY, Sladkevicius P, Kelly S, Ojha K, Campbell S, Narqund G. Evaluation of endometrial receptivity during in-vitro fertilization using three-dimensional power Doppler ultrasound. Ultrasound Obstet Gynecol. 2005;26:765-769. doi: 10.1002/uog.2628. 